\title{
A contemporary view of MDMA
}

\author{
R. De La Garza II • K. A. Miczek
}

Published online: 10 November 2006

(C) Springer-Verlag 2006

Several years of pre-clinical and clinical research specify that MDMA (a principal ingredient in the drug known as "ecstasy") is neurotoxic to serotonergic nerve terminals, and these neurobiological changes have been predicted to account for neurocognitive deficits, depression, and anxiety seen in human users. In 2002, a high-profile scientific report presented evidence that acute MDMA exposure caused significant damage to dopamine neurons in nonhuman primates (Ricaurte et al. 2002). The finding received considerable media attention, and gave the public the impression that MDMA was far more hazardous than originally thought. Less than a year later, the authors conceded that they were unable to replicate or extend their findings, and this was attributed to the fact that methamphetamine, not MDMA, was used in the earlier experiment (Ricaurte et al. 2003). Critics heralded the mix-up as the tip of an iceberg of a larger scientific/government coalition with an agenda to push forward the War on Drugs. The battle cry went out that "the US research agency that supports research on drug abuse needs to ensure its independence from intense political pressure to prove that recreational drugs are harmful" (Nature 2003), and a claim was made that NIDA favors "studies that are liable to prove

K. A. Miczek $(\square)$

Department of Psychology, Bacon Hall, Tufts University, 530 Boston Avenue,

Medford, MA 02155, USA

e-mail: klaus.miczek@tufts.edu

R. De La Garza II

Department of Psychiatry and Biobehavioral Sciences,

David Geffen School of Medicine at The University of California

at Los Angeles,

Los Angeles, CA, USA

e-mail: rdlgarza@mednet.ucla.edu the toxicity of recreational drugs" (Knight 2003). As expected, this issue provided fodder for individuals and organizations that would like a major revamping of MDMA research efforts.

As a result, a debate on the actual harms of MDMA use was ignited with questions on whether MDMA is neurotoxic at all (Morris 2003). In the following year, Rich De La Garza and Ron Cowan organized a mini-symposium for the annual meeting of the Society for Neuroscience with the intent of providing the essential facts of neurotoxicity and neurocognitive changes that result from MDMA exposure in humans and animals. The current special issue is one outcome of that presentation and the contributions include original investigations as well as reviews that provide a critical analysis of published data pointing out strengths and weaknesses of established findings and frequently used methodologies.

The special issue begins with the elegant and comprehensive work of Baumann and colleagues who show that typical doses of MDMA causing long-term serotonin (5-HT) depletion in rats do not reliably increase markers of neurotoxic damage such as cell death, silver staining, or reactive gliosis. MDMA administration induces persistent anxiety-like behaviors in the absence of measurable 5-HT deficits. A review article by De La Garza and colleagues reveals that MDMA serves as a reinforcer in rats (as measured in intravenous self-administration assays), though the responses are not of similar frequency relative to those previously reported for other psychostimulants (e.g., cocaine). Kuhn and colleagues report sex-specific differences in locomotor effects produced by MDMA. Female rats exhibit greater behavioral activation than males after MDMA treatment initially and upon challenge after a 2 -week recovery period. The mechanism by which MDMA increases locomotor activity more in females is attributed to 
gonadal steroid effects on the dopaminergic and serotonergic mechanisms through which MDMA acts.

Fantegrossi reviews the reinforcing effects of MDMA, its enantiomers, and several structural analogs in rhesus monkeys. MDMA and related compounds function as reinforcers, maintaining self-administration behavior greater than that engendered by saline, but less than that engendered by traditional psychostimulants. These findings are remarkable since structurally distinct serotonergic hallucinogen-like drugs (e.g., LSD) do not maintain reliable self-administration in laboratory animals. Of particular interest, during prolonged MDMA self-administration, MDMA-maintained responding progressively weakens, and MDMA eventually fails to maintain significant selfadministration. In a separate and complementary report, Wang and Woolverton compared self-administration of methamphetamine, MDMA, and its isomers using a progressive-ratio schedule in rhesus monkeys. Their data indicate that MDMA and (+)-MDMA were positive reinforcers, but weaker than methamphetamine, while $(-)$-MDMA was, at best, a weak reinforcer in some mon keys. Their data support the hypothesis that increasing 5-HT releasing potency relative to DA is associated with weaker reinforcing effects.

In a report using in vitro cell cultures, Verrico and colleagues investigate the interaction between the different isomers of MDMA and binding to the dopamine transporter (DAT), norepinepherine transporter (NET) and serotonin transporter (SERT) in stably transfected human embryo kidney cells. The thorough and comprehensive series of experiments indicates that the selective neurotoxicity of MDMA on 5-HT neurons cannot be attributed to higher affinity of MDMA for the SERT, compared with the DAT or NET. Of particular interest is the finding that MDMA displayed higher affinity for the human NET and lower affinities for the SERT and DAT which is distinctly different from observations in rat transporters. The effects produced by MDMA are likely the result of its interaction at multiple transporters, autoreceptors, and postsynaptic receptors. In an experimental study with rats, Izco et al. demonstrate that exposure to large ethanol binge-like doses under warm ambient conditions MDMA produces a particularly large loss of 5-HT contentrations and 5-HT transporter density in the hippocampus.

Most articles in the special issue are dedicated to research in humans. Tancer and Johanson found that $1.5 \mathrm{mg} / \mathrm{kg}$ MDMA increased most ratings on the addiction, mood and hallucinogenic scales, and also elevated blood pressure and heart rate in recreational MDMA users. Repeated fluoxetine administrations attenuated the MDMA effects and supported a link of the behavioral effects of
MDMA to brain 5-HT. Roiser and colleagues assessed neuropsychological function in current MDMA users, exMDMA users, polydrug controls, and drug-naive controls using a battery of computerized tests focused on memory, executive function, impulsivity and risk-taking. The results indicate few differences between groups, and on no measure were the current MDMA users impaired significantly relative to the polydrug controls. Some individuals may be particularly vulnerable to cognitive impairment following chronic use, while others remain unaffected. Quednow and colleagues examined the cognitive neurotoxicity of MDMA with regard to behavioral impulsivity and decision-making. Their sample included abstinent, heavy MDMA users, abstinent cannabis users, and drug-naive controls. Heavy use of MDMA may elevate behavioral impulsivity and impair decision-making. Kalechstein and colleagues performed a meta-analysis to determine the association between MDMA misuse and neurocognition. The manuscript offers a number of novel insights and conclusions including the pattern of deficits observed, treatment for neurocognitive impairment in MDMA users, and the relationship between MDMA-associated neurocognitive deficits and functional outcomes. Cowan's report highlights that the current state of neuroimaging in human MDMA users does not permit conclusions regarding the long-term effects of MDMA exposure. He offers suggestions for improvement of future studies, including improved sample homogeneity, increased length of MDMA abstinence, longitudinal study design, test-retest measures, serotonergic specificity, and multimodal approaches. Kuypers and Ramaekers examined whether a single dose of MDMA $(75 \mathrm{mg})$ affects spatial memory performance. Their sample included recreational MDMA users who were treated with placebo, MDMA, and methylphenidate. MDMA, but not methylphenidate, impaired spatial memory for location though processing of contextual information remained intact. The essence of this special issue is captured by Guillot and Berman who provide a Letter to the Editor with evidence challenging the notion that MDMA exposure leads to psychiatric disorders. They rightly specify that scientists have a responsibility to accurately portray the health risks of drug use to policy makers and health care professionals.

In summary, this special issue provides novel data and reviews of the essential facts of neurotoxicity, and neurocognitive and behavioral changes that result from MDMA exposure in humans and animals. The contributions highlight emerging insights into the consequences of shortand long-term MDMA exposure, and the knowledge amassed is expected to increase our understanding of this illicit drug of abuse. 


\section{References}

Knight J (2003) Agony for researchers as mix-up forces retraction of ecstasy study. Nature 425:109

Morris K (2003) Research reawakens ecstasy neurotoxicity debate. Lancet Neurol 2:650
Nature (2003) Ecstasy's after-effects. Nature 425:223

Ricaurte GA, Yuan J, Hatzidimitriou G, Cord BJ, McCann UD (2002) Severe dopaminergic neurotoxicity in primates after a common recreational dose regimen of MDMA ("ecstasy"). Science 297:2260-2263

Ricaurte GA, Yuan J, Hatzidimitriou G, Cord BJ, McCann UD (2003) Retraction. Science 301:1479 